\title{
CMAP and FCMAP Comparisons Using Monte-Carlo Simulations
}

\author{
Paul Cao \\ pcao@ashland.edu, 419-289-5960 \\ Department of Computer Science \\ Ashland University, Ashland OH 44090, USA
}

\begin{abstract}
Biological systems are complex, consisting of many elements of different nature. As a whole, they are robust, and a general system description can be done in a semi-quantitative way when it comes to phenotype behaviors. We used these properties earlier $[1,2]$ to develop two new systems biology methods, causal mapping (CMAP) and Fuzzy CMAP (FCMAP). In this research, a general Monte-Carlo simulation was carried out to compare the behaviors of CMAP and FCMAP in cortical spread cell oscillations. The oscillation period and magnitude from both approaches are compared. The presented development lies on the way to a more general justification of the applicability of FCMAP in real biological system modeling.
\end{abstract}

Keywords: CMAP, FCMAP, Oscillation

\section{Introduction}

The main obstacle of modern theoretical biology is the complexity of objects and explosion of information produced by experimentation. Thus, the development of new theoretical methods to analyze and interpret the wealth of complex data is enormously important [4-5]. Biological processes that occur at the cellular level and consist of large numbers of interacting elements are highly nonlinear, and generally involve multiple time and spatial scales. The quantitative description of these complex systems is of great importance but presents large challenges.

There are many mathematical techniques that have been developed to study network dynamics in a simplified manner. CMAP [1], a new systems biology approach, provides a semi-quantitative description of the dynamic behavior of a network whose elements are causally connected. The efficiency with which the CMAP can be constructed and analyzed makes it an appealing research tool for exploring whether proposed mechanisms are sufficient to describe various motile phenomena. It has the potential to become an intermediate step between a qualitative cell biological hypothesis and full quantitative modeling, permitting cell biologists to partially validate their descriptive models using an intuitive exploratory tool.

Fuzzy sets are adopted to model the biological vagueness and uncertainty in CMAP with a more quantitative representation [2]. Though CMAP uses a graphical formalism to model dynamical processes (see next section for details), the abstraction of information into concepts is the bottleneck on the development of CMAP. Given a biological system, its inherent complex hierarchy structure and

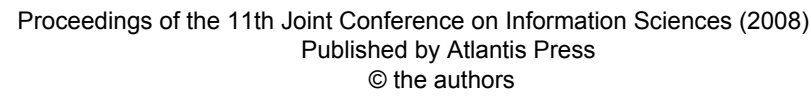


uncertainty can absorb external/internal perturbations in a ubiquitous robust manner. To represent biological robustness features, commonly used quantitative methods, such as differential equations, are not suitable because these approaches ultimately substitute biological complexity with mathematical complexity without showing the fundamental features of the system. Thus, (semi)qualitative methods should be adopted. It should be noted that while these methods are useful in pinpointing the sources of the behavior phenotype they lose the details of the traditional methods. The important rationale behind FCMAP is that, in many cases, cell, tissue, etc. the description is sufficient on the level of linguistic variables, such as 'low' and 'high', 'strong' and 'weak', etc. Fuzzy system is strong in presenting and interpreting vague information, and is employed in this paper to encompass the quantitative and qualitative attributes in CMAP. Our FCMAP approach will allow researchers to study the biological system in a systematic manner at various levels of organization and to apprehend the mechanisms of the formation of system's phenotype at the same time. The detailed description of CMAP and FCMAP as well mathematical equations can be found in $[1,2]$.

\section{Motivation}

The reasons why CMAP and FCMAP have to be compared are the following: first, FCMAP encompasses fuzzy uncertainty in membership functions and it is supposed to behave at least as good as CMAP in a general setting. Secondly, the practical computation of FCMAP is much slower than CMAP. So without a sound justification from real simulations, the conclusion about the advantage of FCMAP is not well supported. Last but least, the simulations of both settings may well benefit the future design of causal mapping because we will obtain more dynamic properties in our simulation.

As a case study we used the phenomenon of contractility oscillations in spreading cells in which microtubules have been depolymerized. How the complex mechano-chemical system governs the oscillations is not known precisely. A cell biological hypothesis was proposed in the original work [3] to explain the data. This hypothesis was further developed and tested by a CMAP [1]. The CMAP (Fig.1) suggested that the source of the oscillations results from the opposing effects of Rho activation leading to a decreased level of myosin light chain phosphatase and a cyclic calcium influx caused by increased membrane tension and leading to a periodically enhanced activation of myosin light chain kinase. The key in the CMAP is a negative feedback from contractility to SAC without which no oscillation would be possible. The concept "Ca-pump" in Fig. 1 is presented for convenience, but it does not have influence in this CMAP: instead the $\left[\mathrm{Ca}^{2+}\right]_{\mathrm{i}}$ decrease due to pumping and association with CaM is modeled as a negative self-influence depicted as a red loop around the calcium concept. CMAP also explicitly depicts all the influences between the elements (concepts). For example, the scheme does not show the influence of the complex $\mathrm{Ca}-\mathrm{CaM}$ on $\mathrm{Ca}$ while the dissociation of the former will free calcium increasing its amount and, at the same time, will decrease the amount of this complex. Our modeling reproduced experimental data, and made a few key predictions. In our CMAP (Fig.1), we showed that no oscillation of the level of MLC-phosphatase is needed to produce

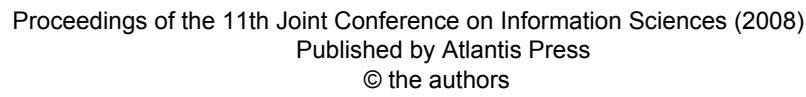


cortical oscillations. The validity of this assumption can be tested using appropriate biosensors. The experimental data also demonstrated that the oscillations were completely damped when the RhoROCK pathway is blocked [3], presumably giving a rise to high level of MLCphosphatase that in turn strongly suppressed the phosphorylated myosin light chain and reduced contractility. Indeed, when we increased the level of MLCphosphatase, our simulation reproduced this property. Oscillations can also be damped by reducing the level of MLCphosphatase. Therefore, we predicted an existence of an optimal range of MLCphosphatase activity, as regulated in part by Rho levels, in which the oscillatory phenotype will occur.

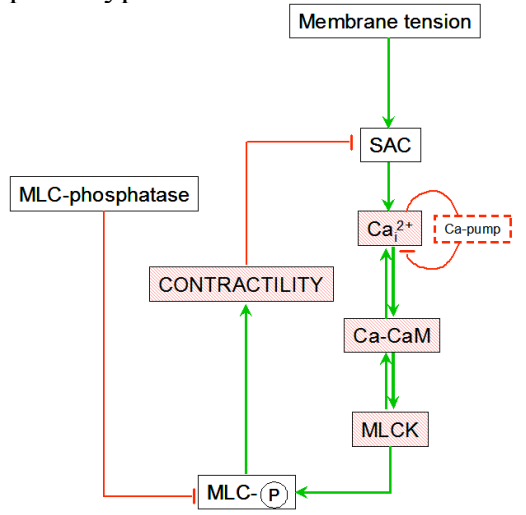

Figure 1. CMAP representation of the hypothesis for cortical cell oscillations. Note that each concept can change only if there is an input depicted as connector directed to this concept. Self-influences are depicted by shadowed boxes for simplicity of representation.

\section{Methodology}

The simulation on F-CMAP involves the changes of SAC-Ca weight. In the original CMAP experiments, we had streptomycin in the buffer. It is known to block the SAC. Our repeated experiments did not include streptomycin and it turns out that the cells still oscillate and the pe- riod is about $20 \mathrm{sec}$ longer. Our hypothesis is that in the previous experiment, not all SACs are blocked so there are still enough to produce the effect. In the second case there are even more SACs and obviously it adds to the effect. As an initial comparison between CMAP and FCMAP in this context, a Monte-Carlo simulation was performed to investigate the difference of their behaviors. Both approaches use the same configuration as shown in Fig 1 and 500,000 sets of weights were randomly generated excluding the weight from SAC to $\mathrm{Ca}^{2+}$. Then the SAC-Ca weigh was increased from 0.1 to 0.9 with 0.2 as a step interval, i.e. 5 levels for the weight [2]. Out of the half million weights, there are 39 sets that produced oscillations in both CMAP and FCMAP. If the SAC-Ca weight is considered, there are 79 sets of weights that produced oscillation. We are particularly interested in the oscillation period and the magnitude in the two types of MAPs given the same set of weights. Statistically, the oscillation period of CMAP is larger than that of the FCMAP (p-value less than $10^{-5}$ in t-test). However, the oscillation magnitudes of the two configurations are statistically the same ( $p$-value $=$ .3595 in the Wilcoxon-Mann-Whitney test). Fig. 2 gives the boxplots and histograms of oscillation periods and magnitudes in the Monte-Carlo Simulation. Though in general, FCMAP has a smaller oscillation period, we observed that if the original weights is fixed as used in [1] and the weight from SAC to $\mathrm{Ca}$ is increased from .1 to .9, FCMAP did produced oscillations with decreasing periods (i.e. our experiment result is matched) while oscillation in CMAP failed to report this key change. However,

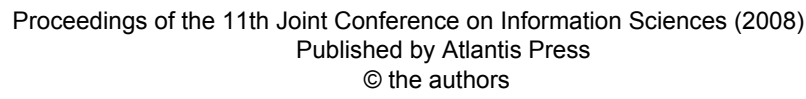


this phenomenon may be due to chance thus more simulations in this aspect will performed.
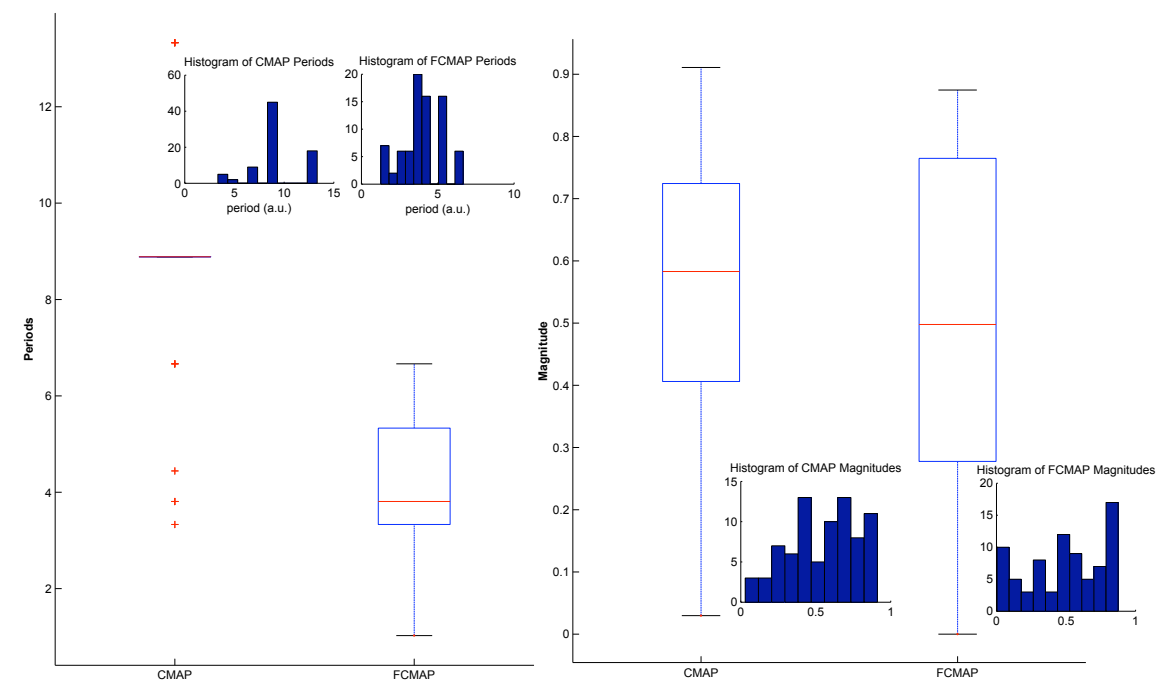

Fig.2 The comparison of oscillating magnitudes and periods in CMAP and FCMAP.

Cortical Oscillations in Spreading

\section{Conclusion}

This paper presents the preliminary research results in comparing CMAP and FCMAP in the cortical spread cell oscillations. The results show that the oscillation periods of FCMAP are statistically shorter than CMAP in general but the magnitudes of the two approaches show no difference.

Future work concerning FCMAP will be theoretically deductions on reasons why the different periods are observed. More simulations will also be carried out to confirm our current hypotheses.

[1] G.E. Weinreb, T.C. Elston, and K. Jacobson, "The Causal Map as a Tool to Mechanistically Interpret Phenomena in Cell Motility: Application to
Cells". Cell Motility and Cytosceleton, 2006. 63(9): p. 523-532

[2] G.E. Weinreb and P. Cao, "Fuzzy causal mapping (F-CMAP) - a proposal to develop a new systems biology tool." Proc. of $10^{\text {th }}$ Joint Conference on Information Science. pp. 130137, 2007

[3] O. Pletjushkina, et al., "Induction of cortical oscillations in spreading cells by depolymerization of microtubules". Cell Motility and the Cytoskeleton, 2001. 48: p. 235-44.

[4] H. Kitano, "Systems biology: A brief overview". Science, 2002. 295(5560): p. 1662-1664.

[5] H. Kitano, "Computational systems biology". Nature, 2002. 420(6912): p. 206-210. 
[6] J. Gagneur, and G. Casari, "From molecular networks to qualitative cell behavior". Febs Letters, 2005. 579(8): p. 1867-1871.

Proceedings of the 11th Joint Conference on Information Sciences (2008)

Published by Atlantis Press (C) the authors 